\title{
BASIC SCIENCE ARTICLE Differential effects of Clostridium difficile toxins on ion secretion and cell integrity in human intestinal cells
}

\author{
Vittoria Buccigrossi ${ }^{1}$, Andrea Lo Vecchio ${ }^{1}$, Antonella Marano ${ }^{1}$ and Alfredo Guarino ${ }^{1}$
}

BACKGROUND: Toxin A (TcdA), toxin B (TcdB), and binary toxin (CDT) produced by Clostridium difficile (CD) are thought to play a key role in inducing diarrhea. The aim of this study was to investigate the individual and combined roles of CD toxins in inducing enterotoxic and cytotoxic effect.

METHODS: Ion secretion and epithelial damage were evaluated in the Ussing chambers as measure of enterotoxic or cytotoxic effect, respectively, in human-derived intestinal cells.

RESULTS: When added to the mucosal side of Caco- 2 cells, TcdB, but not TcdA, induced ion secretion and its effects increased in the presence of TcdA. CDT also induced ion secretion when added to either the mucosal or serosal compartment. Serosal addition of TcdB induced epithelial damage consistent with its cytotoxic effect. However, mucosal addition of TcdB had similar effects, but only in the presence of TcdA. CDT induced epithelial damage when added to the serosal side of cell monolayers, and this was associated with a late onset but prolonged effect. All data were replicated using human colon biopsies.

CONCLUSIONS: These data indicate that $C D$, through the combined and direct activity of its three toxins, induces integrated and synergic enterotoxic and cytotoxic effects on the intestinal epithelium.

Pediatric Research (2019) 85:1048-1054; https://doi.org/10.1038/s41390-019-0365-0

\section{INTRODUCTION}

Several enteric pathogens induce diarrhea via the effects of enterotoxins, which directly increase $\mathrm{Cl}^{-}$secretion and reduce $\mathrm{Na}^{+}$absorption by acting on apical membrane transporters or the lateral spaces between cells that are regulated by tight junctions. Bacterial enterotoxins activate signaling molecules such as cyclic AMP, cyclic GMP, or intracellular $\mathrm{Ca}^{2+}$, which open cellular $\mathrm{Cl}^{-}$ channels, leading to an increase in $\mathrm{Cl}^{-}$and water secretion. ${ }^{1}$ Other pathogens induce cell damage by targeting the enterocyte cytoskeletal network, which is directly implicated in paracellular fluid absorption. Structural damage is induced by the direct invasion of enterocytes by enteric microorganisms or by the effects of cytotoxins.

The loss of barrier function, as a consequence of tight junction disruption, impairs vectoral ion absorption and secretion by the intestinal epithelium. Diarrhea is therefore the result of enterotoxins that induce net fluid secretion into the intestinal lumen (secretory diarrhea) and/or cytotoxins that induce epithelial damage reducing the digestive-absorptive surface. This impairs nutrient absorption and causes osmotic diarrhea. Specific enteric pathogens act through one or more mechanisms, whereas others such as Rotavirus induce diarrhea through a combination of mechanisms. ${ }^{2}$ The enterotoxic (secretory) and the cytotoxic (epithelial damage) mechanisms have clinical manifestations of dehydration and nutrient malabsorption, respectively.

Clostridium difficile (CD) is a sporogenic, anaerobic, Grampositive organism that is responsible for a broad spectrum of diseases in humans, ranging from self-limiting secretory diarrhea to life-threatening conditions such as pseudomembranous colitis, toxic megacolon, intestinal perforation, septic shock, and death. ${ }^{3}$ Severe clinical features are more frequent in at-risk subjects including elderly individuals and those with underlying chronic conditions.

Over the past decade, a dramatic increase in the incidence and severity of $C D$ has been reported worldwide also due to a large misuse of antibiotics and the increase in the use of other therapies that enhance microbiota disruption and promote the emergence of hypervirulent strains. ${ }^{4-6}$

During infection, $C D$ can produce three virulence toxins, namely toxin $A(T c d A)$, toxin $B(T c d B)$, and a binary toxin (CDT); however, their role in the pathophysiology of diarrhea is unclear. ${ }^{7}$ The pathogenesis of $C D$ is strongly related to the activity of $\operatorname{Tcd} A$ and TcdB, two glycosyltransferases that target host GTPases, causing diarrhea and severe colonic inflammation. Their receptors have been described, ${ }^{8}$ but the two toxins are associated with unusual translocation pathways including receptor binding, endocytosis, and pore formation. ${ }^{9}$

Currently, the effects of CD toxins on intestinal cells and their link with clinical symptoms are unclear. One preliminary study indicated that TcdA induces symptoms of CD infection (CDI), whereas TcdB can only induce symptoms in the presence of TcdA or prior damage to the gut mucosa. ${ }^{10}$ Subsequently, it was observed that TcdB is essential for $C D$ virulence and that a strain producing only TcdA lacks virulence. ${ }^{11,12}$ This has created a paradox regarding the individual roles of $\operatorname{TcdA}$ and TcdB.

The Clostridia Research Group demonstrated that production of $\mathrm{TcdB}$ alone was sufficient to cause fatal illness in a hamster model of CDI. ${ }^{11,13}$ They used a gene knockout model to create three stable isogenic mutants of the $C D$ strain, namely $A^{-} B^{+}, A^{+} B^{-}$, and $A^{-} B^{-}$, and found that hamsters receiving the $A^{+} B^{+}$strain, $A^{-} B^{+}$ mutant, or $A^{+} B^{-}$mutant, but not the $A^{-} B^{-}$variant, developed

${ }^{1}$ Department of Translational Medical Science, Section of Pediatrics, University of Naples Federico II, Naples, Italy

Correspondence: Alfredo Guarino (alfguari@unina.it)

Received: 8 October 2018 Revised: 4 February 2019 Accepted: 27 February 2019

Published online: 9 March 2019 
symptoms of $\mathrm{CDI}$. This finding explains many clinical cases of $\mathrm{CDI}$ that can be attributed to $A^{-} B^{+}$strains. ${ }^{14}$

However, both toxins ( $T c d A$ and $T c d B$ ) can cause extensive colonic inflammation and epithelial tissue damage in the infected host via several intracellular pathways, leading to cytoskeletal disruption and apoptosis in enterocytes and immune cells. ${ }^{15,16}$

The other major pathogenic factor of this bacterial species is CDT. This toxin is associated with severe clinical cases, although its role remains unclear. CDT belongs to the binary ADP-ribosylating family of toxins and consists of two components, an enzymatic component (CDTa) and a binding/translocation component (CDTb). CDT leads to the complete destruction of the actin cytoskeleton and ultimately to cell death ${ }^{17,18}$ through lipolysisstimulated lipoprotein receptor (LSR) binding. ${ }^{19}$

Despite the aforementioned research, the effects of individual $C D$ toxins on transepithelial ion transport and cell structure are unknown. Here, we investigated the effects of $C D$ toxins on enterocytes using an in vitro model of ion intestinal transport and epithelial integrity based on signal transduction derived from Ohm's law. ${ }^{20}$ More specifically, we aimed to explore the effects, timing, and site of action of the three $C D$ toxins $(T c d A, T c d B$, and CDT) with respect to the induction of enterotoxic and cytotoxic effects.

\section{METHODS}

Cell culture and chemicals

Caco-2 cells were obtained from the American Type Tissue Culture Collection (Rockville, MD, USA). Cells were grown in Dulbecco's modified Eagle's medium (DMEM Gibco, Grand Island, NY, USA) with a high glucose concentration $(4.5 \mathrm{~g} / \mathrm{L})$ supplemented with $10 \%$ fetal bovine serum, $1 \%$ non-essential amino acids, penicillin $(50 \mathrm{mU} / \mathrm{mL})$, and streptomycin $(50 \mathrm{mg} / \mathrm{mL})$, and maintained in $5 \%$ $\mathrm{CO}_{2} / 95 \%$ air. Cells were used between the 20th and 40th passages, and the medium was changed daily. All chemicals were of reagent grade and were obtained from Sigma Chemicals Co. (St. Louis, MO, USA), unless otherwise stated.

\section{Clostridium difficile toxins}

TcdA, TcdB, and CDT were obtained from List Biological Laboratories (List Lab Inc., CA, USA). One hundred microgram of TcdA was reconstituted with $0.5 \mathrm{~mL}$ of water; the buffer contained $50 \mathrm{mM}$ Tris, $50 \mathrm{mM} \mathrm{NaCl}, \mathrm{pH} 7.5$, and $0.1 \%$ Trehalose. Each vial of TcdB contained $50 \mu \mathrm{g}$ of toxin in $50 \mathrm{mM}$ Tris, $100 \mathrm{mM} \mathrm{NaCl}, \mathrm{pH} 7.5$, at a concentration of $0.2 \mathrm{mg} / \mathrm{mL}$. When examined on $3-8 \%$ sodium dodecyl sulfate polyacrylamide-gel electrophoresis, these proteins migrate as a single major band with an apparent molecular weight of $\sim 300,000 \mathrm{Da}$ for TcdA and 270,000 Da for TcdB.

Densitometric analysis estimates the purity of these toxins as $>80 \% \mathrm{TcdA}$ and $\sim 75 \% \mathrm{TcdB}$ as indicated in data sheet.

Either CDTa or CDTb were reconstituted with $0.1 \mathrm{~mL}$ of water, and the buffer concentration was 20 mM HEPES, pH $7.4+1.25 \%$ lactose. Both toxins were recombinantly expressed in Escherichia coli and purified using affinity chromatography.

Densitometric analysis estimates the purity of these toxins as $>95 \%$ CDTa and $80 \%$ CDTb as indicated in data sheet.

After each toxin was tested at different concentrations in the first set of experiments, the effective dose was identified and was used for every following experiment: $67 \mathrm{pM}$ TcdA, $74 \mathrm{pM} \mathrm{TcdB}$, $100 \mathrm{ng} / \mathrm{mL}$ CDTa +200 ng/mL CDTb (Supplementary Tables).

\section{Ion transport studies}

Cells were grown on uncoated polycarbonate transwell filters from Costar (Costar Italia, Milan, Italy) and used for intestinal transport studies at 15 days post confluence. The experiments were performed in the Ussing chambers (Physiological Instruments, San Diego, USA). The following electrical parameters were measured at different time points after infection: short-circuit current (Isc) is expressed in $\mu \mathrm{A} / \mathrm{cm}^{2}$, transepithelial potential difference (PD) in $\mathrm{mV}$, and tissue ionic conductance $(G)$ in $\mathrm{mS} / \mathrm{cm}^{2}$. lon secretion was studied by monitoring Isc, as an indication of active, luminally directed anion secretion. Maximal changes in short-circuit current $(\Delta \mathrm{I} s)$ were recorded as an indicator of mucosal ion secretion. Cell viability was evaluated at the end of each experiment by the absence of significant tissue ionic conductance loss ( $<20 \%$ vs. basal value) and by measuring the electrical response to the serosal addition of theophylline $(5 \mathrm{mM})$. $P D$, Isc, and $G$ were monitored before and after mucosal or serosal addition of $C D$ toxins. Caco- 2 cell monolayers were incubated 30 min at $37^{\circ} \mathrm{C}$ with $\mathrm{CD}$ toxins, and then were mounted in the Ussing chamber. CFTR(inh)-172 (10 $\mu \mathrm{M}$, Sigma-Aldrich SRL, Italy) was used to block the specific activity of CFTR to evaluate its involvement on the enterotoxic effects. Cystic Fibrosis Transmembrane Conductance Regulator (CFTR) is a membrane chloride channel located on the apical membrane of enterocytes. A specific inhibitor, CFTR(inh)-172, was added to the mucosal side $15 \mathrm{~min}$ after mounting cell monolayers in the Ussing chambers. To explore the calcium-dependent pathway, CaCCinh-A01 $(10 \mu \mathrm{M}$, Sigma-Aldrich SRL, Italy) was used to block the calcium-dependent chloride channel and was added to the mucosal side 5 min after mounting cell monolayers in the Ussing chambers. Bapta $(20 \mu \mathrm{M}$, Enzo Life Sciences, Inc., NY, USA) was used as a calcium chelator. Caco- 2 cells were pretreated with BAPTA/AM on both the mucosal and serosal sides for $30 \mathrm{~min}$, and then cells were stimulated with CD toxins.

Thapsigargin ( $1 \mu \mathrm{M}$, Enzo Life Sciences, Inc., NY, USA) was used as an inducer of calcium store depletion. Thapsigargin was added to the mucosal and serosal side 20 min before stimulation with $C D$ toxins.

To confirm the results observed in Caco-2 cells, colonic biopsies from four children were mounted in the Ussing chambers and stimulated with CD toxins, and then the Isc was measured. All biopsies were from macroscopically normal areas, and intestinal histology was subsequently reported to be normal. Tissue samples were transported to the laboratory in the culture medium and processed within $1 \mathrm{~h}$. Specimens were washed and observed by stereomicroscope to exclude tissue necrosis. Organ culture was performed in DMEM with a high glucose concentration $(4.5 \mathrm{~g} / \mathrm{L})$ supplemented with $0.5 \%$ fetal calf serum, $1 \%$ non-essential amino acids, $2 \%$ penicillin $(50 \mathrm{mU} / \mathrm{mL})$, and streptomycin $(50 \mathrm{mg} / \mathrm{mL})$, and incubated in $5 \% \mathrm{CO}_{2} / 95 \%$ air for $30 \mathrm{~min}$ with $\mathrm{CD}$ toxins.

Informed consent was obtained from the children's parents. The study protocol was approved by the Ethics Committee of the School of Medicine, University of Naples Federico II, Italy (no. 48/08).

Intracellular concentrations of cyclic nucleotides Intracellular concentrations of CAMP and CGMP were measured with commercial immunoassay kits (BioVision, Inc., CA, USA) according to the manufacturer's instructions. Results were calculated as picomoles of cyclic nucleotides per square centimeter and expressed as the fold increase over the basal level. Cholera toxin $\left(6 \times 10^{-8} \mathrm{M}\right)$ and $E$. coli heat-stable enterotoxin (ST) $\left(1 \times 10^{-6} \mathrm{M}\right)$ were used as positive controls for cAMP and cGMP stimulation, respectively.

Transepithelial electrical resistance measurements

Transepithelial electrical resistance (TEER) of cell monolayers grown on filters was measured using a Millicel-ERS resistance monitoring apparatus (Millipore). The net TEER (in Ohms $/ \mathrm{cm}^{2}$ ) was calculated by subtracting the background from the actual value and multiplying this value by the filter area $\left(4.9 \mathrm{~cm}^{2}\right)$.

Statistical analysis

Each experiment was performed in triplicate and repeated at least three times. Results are expressed as the fold increase \pm SD vs. 
basal conditions. Results were considered significant at $p<0.05$. The GraphPad Prism Software (San Diego, CA, USA) was used to perform two-tailed, unpaired Student's $t$ tests and two-tailed, paired Student's $t$ tests to evaluate statistical significance. The $p$ value for each analysis is indicated in the figure legends.

\section{RESULTS}

Effects of CD toxins on transepithelial ion transport in intestinal epithelial cells

$\mathrm{TcdA}, \mathrm{TcdB}$, and CDT, alone or in combination, were added to Caco- 2 cell monolayers, and the Isc was monitored as described in the Methods section. In the first set of experiments, each toxin was tested at different concentrations to identify the effective dose (Supplementary Tables). Titration experiments were performed using Isc as a marker of potency with respect to the induction of ion secretion; the maximal effective dose and site of action for CD toxins are indicated in Supplementary Tables 1-3.

TcdA alone did not affect Isc (Fig. 1a, b). In contrast, TcdB was effective in increasing Isc and this effect was increased when Caco-2 cell monolayers were pre-incubated with TcdA (Fig. 1a, b). Further, the site of action of TcdB was found to be the mucosal compartment (Supplementary Tables 1, 2). CDT induced an increase in Isc when added either to the mucosal or the serosal side of Caco-2 cells and its potency was similar to that of TcdA plus TcdB (Supplementary Table 3; Fig. 1b). For all CD toxins, the Isc response was observed $\sim 20$ min after toxin addition and reached a plateau after $\sim 60 \mathrm{~min}$. The Isc increase was sustained for the entire duration of the experiments. The time course of the effects of toxins is shown in Fig. 1a.

To validate the data obtained from Caco- 2 cells, CD toxins were used to stimulate human colonic biopsies mounted in the Ussing chambers. Colonic biopsies from four children were mounted in the Ussing chambers, stimulated with CD toxins and Isc was measured. Isc significantly increased upon TcdB and CDT, confirming the secretive pattern observed in Caco-2 cells (Fig. 1c).

Specific molecular pathways involved in the effects of $C D$ toxin To investigate the specific pathways involved in the effects of $C D$ toxins, we studied the role of CFTR, cyclic nucleotides, and calcium in the observed chloride secretion. The CFTR inhibitor CFTR(inh)172 was used for intestinal ion transport studies using the Ussing chambers; in its presence, the enterotoxic effects induced by the combination of $T c d A / T c d B$ and CDT were inhibited, suggesting that this specific chloride channel is involved in CD toxin-induced chloride secretion (Fig. 2a).

Because CFTR can be activated either by CAMP or CGMP, we measured the intracellular concentrations of these second messengers upon $C D$ toxin stimulation. Levels of both CAMP and cGMP were increased compared to those in basal controls, but the response pattern depended on the specific $C D$ toxin. The combination of $\mathrm{TcdA} / \mathrm{TcdB}$ and $\mathrm{CDT}$ induced a mild increase in CAMP, compared to that in controls and that with cholera toxin, the classic bacterial enterotoxin that functions via the cAMP pathway (Fig. 2b). Further, intracellular levels of cGMP were significantly increased by CD toxins (Fig. 2C). Thus, these data suggest that $C D$ toxins induce ion secretion mainly via the cGMP pathway, although the CGMP increase did not reach the magnitude observed with $E$. coli ST.

Bacterial toxins can induce diarrhea through a calciumdependent pathway. To assess the role of intracellular calcium in toxin-induced secretion, we used a specific inhibitor of the CaCC channel (CaCCinh-172) and a calcium chelator (BAPTA). With these inhibitors, no differences were observed in Isc compared to that with standard conditions, indicating that calcium-dependent pathways are not involved in the enterotoxic effects of CD toxins. To further investigate the role of the calcium pathway, thapsigargin was used to deplete the intracellular calcium stores before
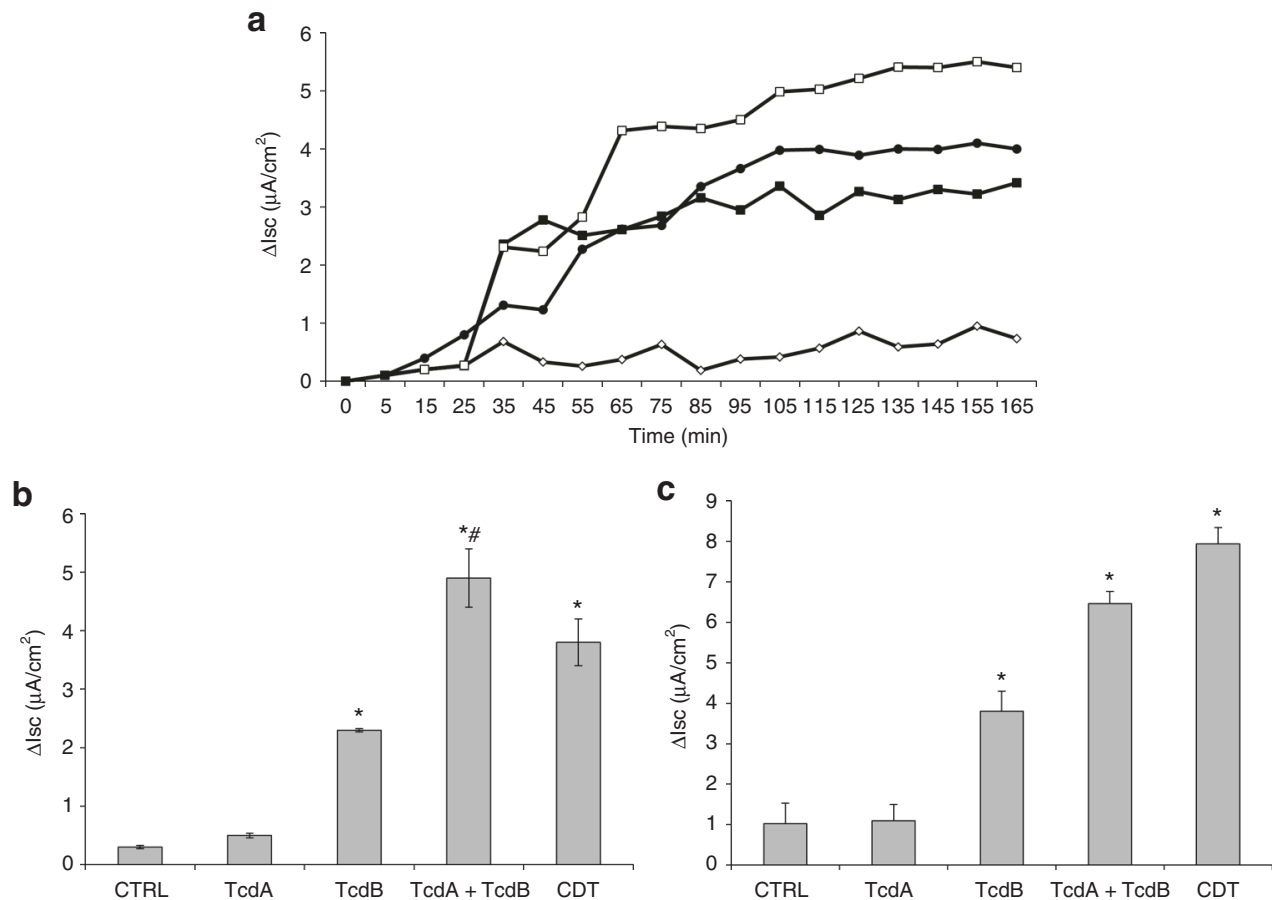

Fig. 1 Effects of Clostridium difficile toxins (CD) on chloride secretion in intestinal epithelium. a Representative experiment showing the time course of short-circuit current (Isc) modifications induced by CD toxins, alone or in combination, in Caco-2 cell monolayers; toxin A (TcdA) $(67 \mathrm{pM})(())$, toxin B (TcdB) $(74 \mathrm{pM})(\square)$, TcdA + TcdB $(67+74 \mathrm{pM})(\square)$, and binary toxin (CDT) $(2.08 \mathrm{nM}$ CDTa $+2.7 \mathrm{nM}$ CDTb) $(\mathbf{})$ stimulated cells. b Caco-2 cell monolayers were mounted in the Ussing chambers, stimulated with CD toxins (TcdA 67 pM, TcdB 74pM, 2.08 nM CDTa + $2.7 \mathrm{nM}$ CDTb) at the mucosal side and Isc was measured. ${ }^{*} p<0.05$ vs. CTRL. ${ }^{*} p<0.05$ vs. TcdB. c Human colonic biopsies were mounted in the Ussing chambers, stimulated with CD toxins (TcdA $67 \mathrm{pM}$, TcdB 74pM, $2.08 \mathrm{nM}$ CDTa $+2.7 \mathrm{nM}$ CDTb) and Isc was measured. ${ }^{*} p<0.05$ vs. CTRL 

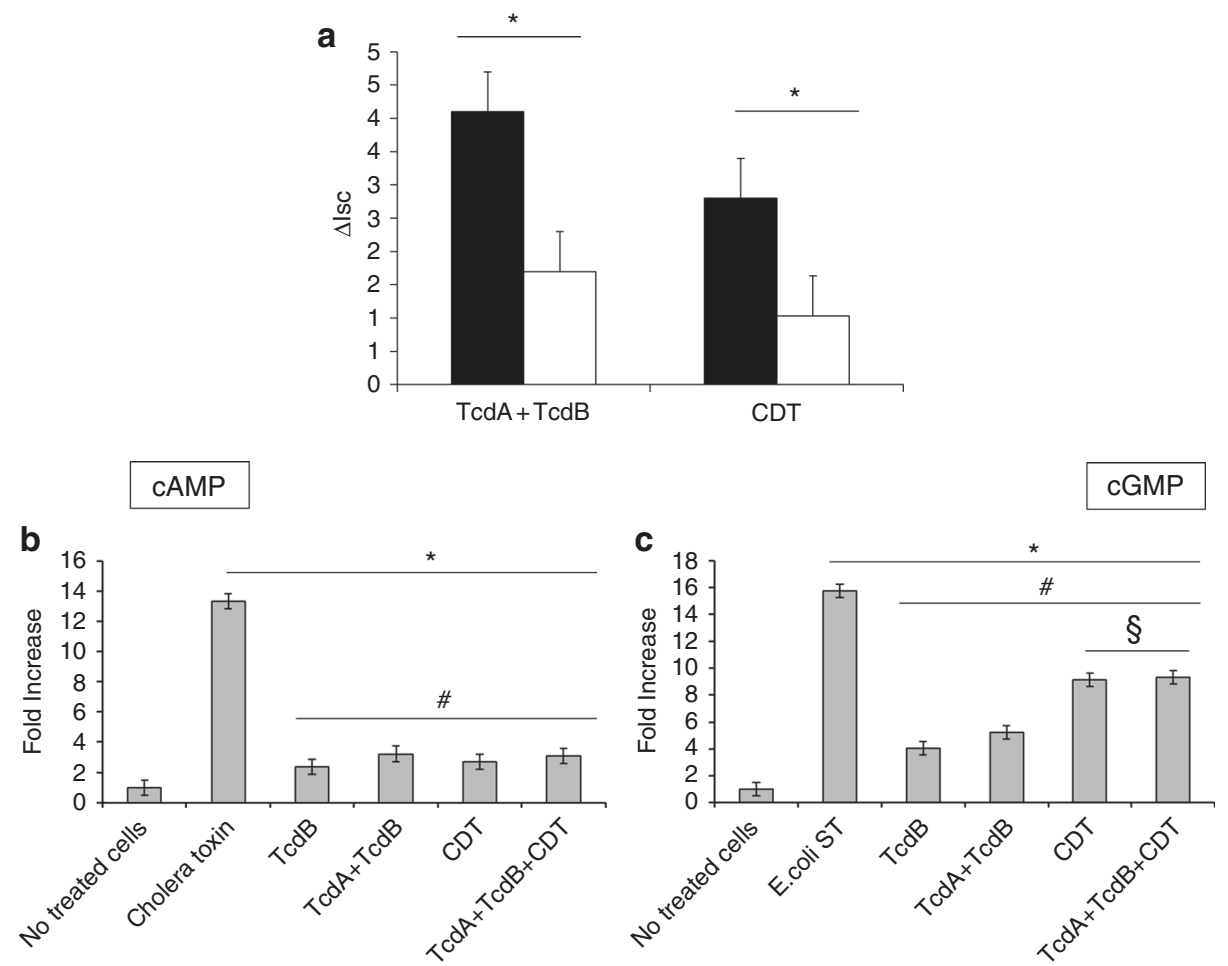

Fig. 2 Involvement of specific molecular pathways affected by Clostridium difficile (CD) toxins. a Isc was significantly reduced in the presence of CFTR inhibitor (white bar) compared with standard conditions (black bar), indicating that the enterotoxic effects induced by CD toxins is mediated by CFTR activity. ${ }^{*} p<0.05$ vs. standard condition. $\mathbf{b}$ Effect of CD toxins on CAMP intracellular levels. Cholera toxin $\left(6 \times 10^{-8} \mathrm{M}\right)$ was used as a positive control. ${ }^{*} p<0.05$ vs. no treated cells; ${ }^{*} p<0.01$ vs. Cholera toxin. $c$ Effect of CD toxins on cGMP intracellular levels. Escherichia coli ST $\left(1 \times 10^{-6} \mathrm{M}\right)$ was used as a positive control. ${ }^{*} p<0.05$ vs. no treated cells; ${ }^{*} p<0.05$ vs. E. coli ST; ${ }^{\S} p<0.05$ vs. toxin B (TcdB) or toxin B $(\mathrm{TcdA})+\mathrm{TcdB}$

$C D$ toxin stimulation. Again, the effects of $C D$ toxins were not modified, further ruling out the involvement of calcium in the observed enterotoxic effects.

Effects of CD toxins on intestinal epithelial integrity

To investigate the effects of $C D$ toxins on intestinal epithelial integrity, we measured TEER after exposing Caco-2 cell monolayers to $C D$ toxins. Initially, we tested $T c d A$ and $T c d B$ separately (Fig. 3a, b). TcdA did not alter resistance when added to the mucosal, serosal, or both sides of the cell monolayers (Fig. 3a). TcdB induced a decrease in TEER when added to the serosal, but not mucosal, side of Caco-2 cell monolayers (Fig. 3b). However, in the presence of $T c d A$, the addition of $T c d B$ to the mucosal side resulted in a decrease in TEER that was not observed in the presence of each toxin alone (Fig. 3c).

Caco- 2 cell monolayers were also stimulated with CDT at the mucosal or serosal side, or both sides, and TEER was monitored after 3 days and then daily for 6 days (Fig. 3d). CDT was effective in inducing epithelial damage, exhibiting a late onset but prolonged effect. In the first 2 days, epithelial resistance did not change in all experimental conditions. Subsequently, starting on day 3, we observed a significant decrease in TEER. However, this was seen when CDT was added to the serosal, but not mucosal, side of cell monolayers. CDT addition to both sides of the Caco-2 cell monolayers induced an earlier decrease in TEER (Fig. 3d).

\section{DISCUSSION}

Although toxigenic $C D$ strains are recognized as pathogenic, the roles and the mechanisms of action of individual toxins are not clear. We investigated the enterotoxic and cytotoxic effects exerted by each toxin, alone or in combination, by analyzing the timing and site of action in polarized intestinal epithelia.
Secretory diarrhea results from altered transepithelial ion transport across the intestinal mucosa and our in vitro model allowed us to study the direct interaction between intestinal epithelial cells and CD toxins. We found that the enterotoxic effect is mainly exerted by $\mathrm{TcdB}$ and CDT. TcdA was effective only in combination with the former, enhancing the TcdB-induced secretory effect. CDT was able to induce chloride secretion and was active on either cell compartment, whereas TcdB was only effective when added to the mucosal side.

The enterotoxic effect was found to be mediated by CFTR activity. Stimulation of colonic CFTR channels involves CAMP- or cGMP-dependent phosphorylation. ${ }^{21}$ Many bacterial enterotoxins stimulate these second messengers ${ }^{22}$ causing electrogenic chloride secretion. ${ }^{23,24}$ Generally, cAMP is responsible for sustained activation of CFTR, whereas CGMP rapidly activates CFTR inducing more potent and rapid, but short-lasting, chloride secretion. Examples of enterotoxins that selectively activate either CAMP or cGMP include cholera toxin and E. coli heat-stable (ST) enterotoxin, respectively. ${ }^{1,25}$

Intracellular calcium provides a third pathway for chloride secretion by enterotoxins. ${ }^{26}$ Our findings showed that $C D$ toxins mainly increase cGMP intracellular levels, with CDT exerting a more potent effect compared to that with TcdA + TcdB. However, the induction of ion secretion is not the only mechanism of action of $C D$ toxins. Several data have indicated that $C D I$ is characterized by intestinal mucosal damage, inflammation, and diarrhea, which are more consistent with a mechanism involving intestinal integrity. ${ }^{27}$

Our data indicated that $T c d B$ and $C D T$ are the main toxins responsible for the cytotoxic effect. TcdA did not alter epithelial integrity in our model, although several authors have suggested that $\operatorname{TcdA}$ is responsible for cytotoxic damage when added to intestinal epithelial cells at high doses. ${ }^{28,29}$ The effect of CD toxins 


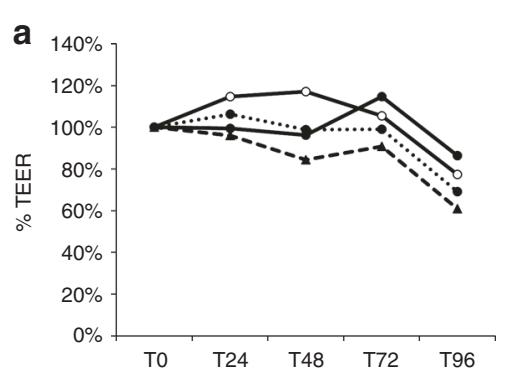

C

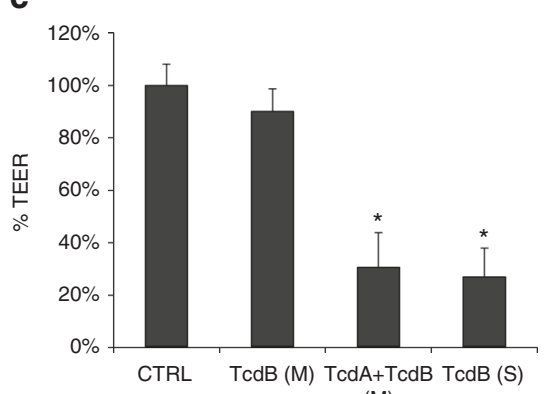

(M)
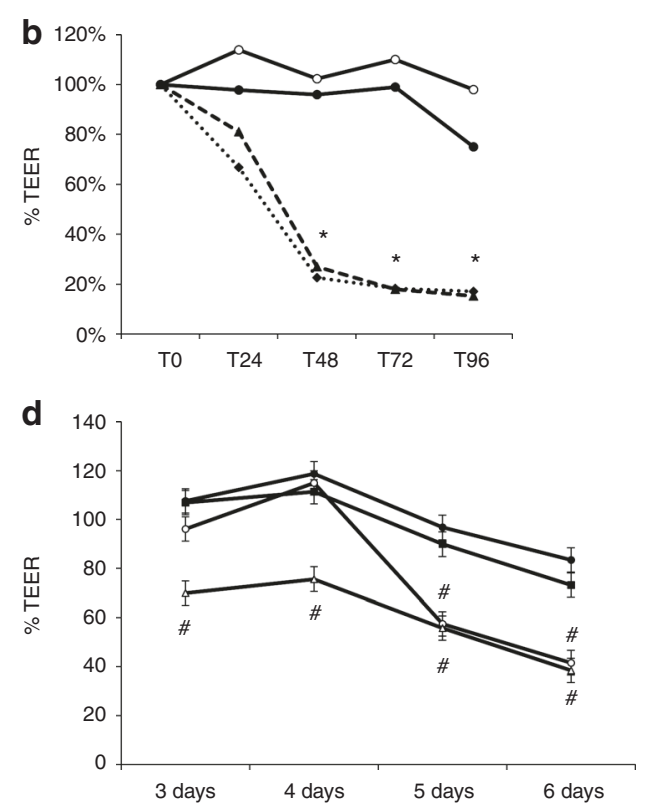

Fig. 3 Effects of Clostridium difficile toxins on intestinal epithelial integrity. Effects of TcdA and TcdB on epithelial integrity. Caco-2 cell monolayers were exposed to TcdA (67 pM) (a) and TcdB (74 pM) (b) and transepithelial electrical resistance (TEER) was measured at 0 (T0), $24 \mathrm{~h}$ (T24), $48 \mathrm{~h}$ (T48), $72 \mathrm{~h}$ (T72), and $96 \mathrm{~h}$ (T96) after toxin stimulation. Site of stimulation: none (O), mucosal side ((), serosal side ( $\mathbf{A})$, mucosal + serosal side $(\diamond) .{ }^{*} p<0.05$ vs. mucosal side. c Caco-2 cells were pre-incubated with TcdA (67pM) at the mucosal side and then TcdB (74pM) was added. ${ }^{*} p<0.05$ vs. CTRL. d Effects of binary toxin (CDT) on epithelial integrity. CDT (2.08 nM CDTa $+2.7 \mathrm{nM}$ CDTb) was added to the mucosal $(O)$, serosal $(O)$, or mucosal and serosal $(\Delta)$ sides of Caco-2 cell monolayers and TEER values were recorded at 3, 4, 5, and 6 days, and compared to those from untreated cells $(\square) .{ }^{\#} p<0.05$ vs. CTRL

was polar, depending on application to the mucosal or serosal compartment and the combination of toxins. Interestingly, TcdB was active at the serosal side, but its effect was only observed in the presence of $\mathrm{TcdA}$, possibly indicating that the latter toxin allows TcdB to reach the basolateral compartment from the intestinal lumen.

CDT was also active at the serosal side, but when it was added simultaneously to both sides, an earlier effect was observed. Timing was also a crucial feature of $C D$ toxins. Whereas the cytotoxic effect of TcdB was evident at 2 days post infection, CDT had a later onset but a prolonged effect. This suggests that $C D$ strains that produce all three toxins $\left(\mathrm{A}^{+} \mathrm{B}^{+} \mathrm{CDT} \mathrm{T}^{+}\right)$fully exploit the synergic enterotoxic and cytotoxic effects exerted by individual toxins on the intestinal epithelium. First, $\mathrm{TcdB}$, in association with TcdA, induces diarrhea through cGMP-dependent chloride secretion, and subsequently impairs the intestinal barrier. CDT stimulates chloride secretion by acting on either the mucosal or serosal side, inducing secretory diarrhea once epithelial integrity is disrupted. These results suggest that intestinal integrity is progressively compromised during the early days of CDI via the effects of TcdB in association with TcdA; subsequently, CDT acts causing prolonged and more severe secretion, in addition to epithelial damage.

These findings are in agreement with studies on $C D$ toxin receptors. Specifically, TcdA and $T c d B$ have different receptors. Sucrase-isomaltase and glycoprotein 96 (gp96) have been identified as TcdA plasma membrane receptors that allow toxin entry into the enterocyte, resulting in the induction of cytotoxicity. ${ }^{30}$ In particular, gp96 is localized to the apical membrane of colonocytes. ${ }^{30}$ In contrast, chondroitin sulfate proteoglycan 4 and the poliovirus receptor-like 3 have been identified as specific TcdB receptors that exhibit no clear localization. ${ }^{31,32}$ Further, the receptor for CDT was recently identified as the $\mathrm{LSR}^{19}$ that clusters in lipid rafts, ${ }^{33}$ which in turn mediates the uptake of CDT and its internalization toward the endosomal compartments. ${ }^{17}$
We hypothesize that $T c d B$ plays a key role in the pathogenesis of $\mathrm{CDI}$ and that TcdA acts enhancing the effects of TcdB. In accordance with our results, a previous study showed that blocking TcdA through the use of specific monoclonal and polyclonal antibodies failed to protect from $\mathrm{CDI}^{34}$ In addition, experiments using isogenic $\operatorname{Tcd} A$ and $T c d B$ mutants of a virulent $C D$ strains in a hamster model showed that $T c d B$ is a key virulence determinant. $^{11}$

From a clinical point of view, there is evidence suggesting a pathogenic role for TcdA-negative/TcdB-positive strains, which have caused more than one outbreak. ${ }^{35,36}$ In addition, in a recent clinical trial, the neutralization of TcdB with monoclonal antibodies was associated with a substantially lower rate of recurrent infections. ${ }^{37}$ This evidence supports our experimental findings of the respective roles of $T c d A$ and $T c d B$.

CDT-producing CD strains such as the epidemic strain NAP1/BI/ 027 are more virulent and associated with severe clinical outcomes. The severe clinical features and high resistance to first-line antibiotic treatments are associated with a higher production of toxins. This feature might, at least in part, be due to the peculiar characteristics of CDT described in our model. The observed intense and prolonged cytotoxic effect could be responsible for severe mucosal damage and intestinal inflammation and, eventually, the induction of bacterial translocation, intestinal perforation, or toxic megacolon.

The clinical context of our results also deserves further discussion. According to clinical practice guidelines for the management of CDI, treatment failure is defined by the "lack of improvement or increasing stool frequency after 3 days of treatment.."138 However, as seen in our experiments, CDT still exerts its effects 5 to 6 days after infection. Therefore, the presence of CDT within the intestinal lumen might be responsible for symptoms that are more prolonged than those expected after effective antibiotic therapy, suggesting that the definition of therapeutic failure might need to be reconsidered. 
Differential effects of Clostridium difficile toxins on ion secretion...

Table 1. Summary of effects of Clostridium difficile toxins on ion transport and epithelial integrity

\begin{tabular}{|c|c|c|}
\hline $\begin{array}{l}\text { Clostridium difficile } \\
\text { toxins }\end{array}$ & Enterotoxic damage & Cytotoxic damage \\
\hline TcdA & $\begin{array}{l}\text { No direct action on chloride secretion (both mucosal and } \\
\text { serosal sides) } \\
\text { TcdA strengthens the effects of TcdB on chloride secretion } \\
\text { when added to the mucosal side }\end{array}$ & $\begin{array}{l}\text { Promotes TcdB activity of reducing epithelial resistance if } \\
\text { exposed to the mucosal side }\end{array}$ \\
\hline $\mathrm{TcdB}$ & $\begin{array}{l}\text { Causes chloride secretion if added to the mucosal side of } \\
\text { the enterocyte }(\sim 1 \mathrm{~h} \text { after exposure) } \\
\text { Chloride secretion is mediated by CFTR channels and } \\
\text { nucleotides (cAMP and cGMP) } \\
\text { Chloride secretion is strengthened if epithelium is pre- } \\
\text { incubated with TcdA }\end{array}$ & $\begin{array}{l}\text { No effect on epithelial resistance if exposed alone to the } \\
\text { mucosal side } \\
\text { Significant reduction in resistance (increase in permeability) if } \\
\text { coupled with TcdA on the mucosal side } \\
\text { Direct effect on epithelial resistance when exposed to the } \\
\text { serosal side }\end{array}$ \\
\hline CDT & $\begin{array}{l}\text { Causes chloride secretion if exposed to the mucosal or } \\
\text { serosal side of the enterocytes } \\
\text { Secretion is partially enhanced by the presence of the other } \\
\text { toxins }\end{array}$ & $\begin{array}{l}\text { Direct effect on epithelial resistance on the mucosal and } \\
\text { serosal sides } \\
\text { Prolonged cytotoxic effects remain evident } 6 \text { days after } \\
\text { infection }\end{array}$ \\
\hline
\end{tabular}

New therapeutic approaches to treat and prevent CDI have been proposed and tested in humans. The use of toxin-targeting monoclonal antibodies is one of the most promising approaches, and has been successfully validated in large clinical trials. ${ }^{37,39,40}$ This immunological approach, based on the direct interaction between $C D$ toxins and the intestinal epithelium, is rapidly changing our knowledge of the pathophysiology of CDI. The inhibition of adhesion to enterocytes and simultaneous blockage of different domain epitopes can neutralize toxin activity, hampering the course of $\mathrm{CDI}$, and preventing its recurrence. ${ }^{41}$ Thus, the proposed use a combined two-phase treatment, with an antibiotic approach and subsequent blockage of CDT toxins via CDT-targeting monoclonal antibodies, should be explored.

In conclusion, the three toxins produced by pathogenic CD strains act in a compartmentalized and integrated mode. TcdB induces ion secretion in intestinal epithelial cells and has an enhanced effect in the presence of the TcdA. CDT also induces chloride secretion. The mucosal side is the main target of the intestinal epithelium, and enterotoxic effects are mainly due to CAMP and CGMP activation of CFTR, whereas the calcium pathway of ion secretion was not found to be involved. The serosal side of the intestinal epithelium was found to be the main target site of $C D$ toxins, which are involved in cytotoxic damage. The mucosal compartment was found to be only targeted by $\mathrm{TcdB}$ in the presence of $\mathrm{TcdA}$, and CDT had no direct effects. These results suggest a pattern regarding the specific effects of $C D$ toxins in their induction of a combination of enterotoxic and cytotoxic damage (Table 1) and explain why hypervirulent strains induce more severe $\mathrm{CDI}$.

\section{AUTHOR CONTRIBUTIONS}

V.B., A.L.V. and A.G. conceived and designed the experiments. V.B. and A.M. conducted the experiments. V.B. and A.L.V. analyzed the data. V.B., A.L.V. and A.G. wrote the paper. All authors edited the draft.

\section{ADDITIONAL INFORMATION}

The online version of this article (https://doi.org/10.1038/s41390-019-0365-0) contains supplementary material, which is available to authorized users.

Competing interests: The authors declare no competing interesta.

Publisher's note: Springer Nature remains neutral with regard to jurisdictional claims in published maps and institutional affiliations.

\section{REFERENCES}

1. Das, S., Jayaratne, R. \& Barrett, K. E. The role of ion transporters in the pathophysiology of infectious diarrhea. Cell. Mol. Gastroenterol. Hepatol. 6, 33-45 (2018).

2. De Marco, G. et al. Rotavirus induces a biphasic enterotoxic and cytotoxic response in human-derived intestinal enterocytes, which is inhibited by human immunoglobulins. J. Infect. Dis. 200, 813-819 (2009).

3. Lo Vecchio, A. \& Zacur, G. M. Clostridium difficile infection: an update on epidemiology, risk factors, and therapeutic options. Curr. Opin. Gastroenterol. 28, 1-9 (2012).

4. Poutanen, S. M. \& Simor, A. E. Clostridium difficile-associated diarrhea in adults. Can. Med Assoc. J. 171, 51-58 (2004).

5. Elliott, B., Chang, B. J., Golledge, C. L. \& Riley, T. V. Clostridium difficile-associated diarrhoea. Intern. Med. J. 37, 561-568 (2007).

6. Furuya-Kanamori, L. et al. Comorbidities, exposure to medications, and the risk of community-acquired Clostridium difficile infection: a systematic review and metaanalysis. Infect. Control Hosp. Epidemiol. 36, 132-141 (2015).

7. Rupnik, M., Wilcox, M. H. \& Gerding, D. N. Clostridium difficile infection: new developments in epidemiology and pathogenesis. Nat. Rev. Microbiol 7, 526-536 (2009).

8. Di Bella, S., Ascenzi, P., Siarakas, S., Petrosillo, N. \& di Masi, A. Clostridium difficile Toxins $A$ and $B$ : insights into pathogenic properties and extraintestinal effects. Toxins (Basel) 8, 134 (2016). http://www.mdpi.com/2072-6651/8/5/134 Accessed 4 Oct 2017.

9. Orrell, K. E., Zhang, Z., Sugiman-Marangos, S. N. \& Melnyk, R. A. Clostridium difficile toxins A and B: receptors, pores, and translocation into cells. Crit. Rev. Biochem. Mol. Biol. 52, 461-473 (2017).

10. Lyerly, D. M., Saum, K. E., MacDonald, D. K. \& Wilkins, T. D. Effects of Clostridium difficile toxins given intragastrically to animals. Infect. Immun. 47, 349-352 (1985).

11. Lyras, D. et al. Toxin B is essential for virulence of Clostridium difficile. Nature $\mathbf{4 5 8}$ 1176-1179, (2009). http://www.pubmedcentral.nih.gov/articlerender.fcgi?artid= 2679968\&tool $=$ pmcentrez\&rendertype $=$ abstract

12. Carter, G. P. et al. Defining the roles of $T c d A$ and $T c d B$ in localized gastrointestinal disease, systemic organ damage, and the host response during Clostridium difficile infections. MBio 6, 1-10 (2015).

13. Kuehne, $\mathrm{S}$. A. et al. The role of toxin A and toxin B in Clostridium difficile infection. Nature 467, 711-713 (2010)

14. Drudy, D., Harnedy, N., Fanning, S., Hannan, M. \& Kyne, L. Emergence and control of fluoroquinolone-resistant, toxin A-negative, toxin B-positive Clostridium diffcile. Infect. Control Hosp. Epidemiol. 28, 932-940 (2007).

15. Carter, G. P., Rood, J. I. \& Lyras, D. The role of toxin A and toxin B in the virulence of Clostridium difficile. Trends Microbiol 20, 21-29 (2012).

16. Chumbler, N. M., Farrow, M. A., Lapierre, L. A., Franklin, J. L. \& Lacy, D. B. Clostridium difficile Toxins $\mathrm{TcdA}$ and $\mathrm{TcdB}$ cause colonic tissue damage by distinct mechanisms. Infect. Immun. 84, 2871-2877 (2016).

17. Gerding, D. N., Johnson, S., Rupnik, M. \& Aktories, K. Clostridium difficile binary toxin CDT: mechanism, epidemiology, and potential clinical importance. Gut Microbes 5, 15-27 (2014).

18. Sundriyal, A., Roberts, A. K., Shone, C. C. \& Acharya, K. R. Structural basis for substrate recognition in the enzymatic component of ADP-ribosyltransferase toxin CDTa from Clostridium difficile. J. Biol. Chem. 284, 28713-28719 (2009). 
19. Hemmasi, S. et al. Interaction of the Clostridium difficile binary toxin CDT and its host cell receptor, lipolysis-stimulated lipoprotein receptor (LSR). J. Biol. Chem. 290, 14031-14044 (2015).

20. Herrmann, J. R. \& Turner, J. R. Beyond Ussing's chambers: contemporary thoughts on integration of transepithelial transport. Am. J. Physiol. Cell Physiol. 310, C423-C431 (2016).

21. Greger, R. Role of CFTR in the colon. Annu Rev. Physiol. 62, 467-491 (2000).

22. Kopic, S. \& Geibel, J. P. Toxin mediated diarrhea in the 21 century: the pathophysiology of intestinal ion transport in the course of ETEC, V. cholerae and rotavirus infection. Toxins (Basel) 2, 2132-2157 (2010).

23. Buccigrossi, V. et al. Polar effects on ion transport and cell proliferation induced by GC-C ligands in intestinal epithelial cells. Pediatr. Res. 69, 17-22 (2011).

24. Canani, R. B. et al. Growth hormone regulates intestinal ion transport through a modulation of the constitutive nitric oxide synthase-nitric oxide-cAMP pathway. World J. Gastroenterol. 12, 4710-4715 (2006).

25. Steinbrecher, K. A. The multiple roles of guanylate cyclase $C$, a heat stable enterotoxin receptor. Curr. Opin. Gastroenterol. 30, 1-6 (2014).

26. Canani, R. B. et al. Effects of HIV-1 Tat protein on ion secretion and on cell proliferation in human intestinal epithelial cells. Gastroenterology 124, 368-376 (2003).

27. $\mathrm{Ng}$, J. et al. Clostridium difficile toxin-induced inflammation and intestinal injury are mediated by the inflammasome. Gastroenterology 139, 542-552.e1-3 (2010).

28. Olling, A. et al. The repetitive oligopeptide sequences modulate cytopathic potency but are not crucial for cellular uptake of Clostridium difficile toxin A. PLoS ONE 6, e17623 (2011).

29. Liu, T. S. et al. Protective role of HSP72 against Clostridium difficile toxin A-induced intestinal epithelial cell dysfunction. AJP Cell Physiol. 284, C1073-C1082 (2003).

30. Na, X., Kim, H., Moyer, M. P., Pothoulakis, C. \& LaMont, J. T. gp96 is a human colonocyte plasma membrane binding protein for Clostridium difficile toxin A. Infect. Immun. 76, 2862-2871 (2008).
31. LaFrance, M. E. et al. Identification of an epithelial cell receptor responsible for Clostridium difficile TcdB-induced cytotoxicity. Proc. Natl Acad. Sci. USA 112, 7073-7078 (2015)

32. Yuan, P. et al. Chondroitin sulfate proteoglycan 4 functions as the cellular receptor for Clostridium difficile toxin B. Cell Res. 25, 157-168, (2015).

33. Papatheodorou, P. et al. Clostridium difficile binary toxin CDT induces clustering of the lipolysis-stimulated lipoprotein receptor into lipid rafts. MBio 4, 1-8 (2013).

34. Steele, J., Mukherjee, J., Parry, N. \& Tzipori, S. Antibody against TcdB, but not $\mathrm{TcdA}$, prevents development of gastrointestinal and systemic Clostridium difficile disease. J. Infect. Dis. 207, 323-330 (2013).

35. Alfa, M. J. et al. Characterization of a toxin A-negative, toxin B-positive strain of Clostridium difficile responsible for a nosocomial outbreak of Clostridium difficileassociated diarrhea. J. Clin. Microbiol 38, 2706-2714 (2000).

36. Kuijper, E. J. et al. Nosocomial outbreak of Clostridium difficile-associated diarrhoea due to a clindamycin-resistant enterotoxin A-negative strain. Eur. J. Clin. Microbiol. Infect. Dis. 20, 528-534 (2001).

37. Wilcox, M. H. et al. Bezlotoxumab for prevention of recurrent Clostridium difficile infection. N. Engl. J. Med 376, 305-317, (2017).

38. Cheng, A. C. et al. Australasian Society for Infectious Diseases guidelines for the diagnosis and treatment of Clostridium difficile infection. Med J. Aust. 194, 353-358 (2011).

39. Lo Vecchio, A., Liguoro, I. \& Scotto, R. Monoclonal antibodies against Clostridium difficile infection in the pipeline. Drugs Fut. 39, 693-700 (2014).

40. Lo Vecchio, A., Della Ventura, B. \& Nicastro, E. Clostridium difficile antibodies: a patent evaluation (WO2013028810). Expert Opin. Ther. Pat. 23, 1635-1640 (2013).

41. Larabee, J. L., Bland, S. J., Hunt, J. J. \& Ballard, J. D. Intrinsic toxin-derived peptides destabilize and inactivate Clostridium difficile TcdB. MBio 8, 1-13 (2017). 